\title{
Unveiling the Chemical Composition of Halide Perovskite Films using Multivariate Statistical Analyses
}

Stefania Cacovich ${ }^{1 \S *}$, Fabio Matteocci ${ }^{2}$, Mojtaba Abdi-Jalebi ${ }^{3}$, Samuel D. Stranks ${ }^{3}$, Aldo Di Carlo $^{2}$, Caterina Ducati ${ }^{1}$, Giorgio Divitini ${ }^{l}$ *.

${ }^{1}$ Department of Materials Science and Metallurgy, University of Cambridge, 27 Charles Babbage Road, Cambridge CB3 OFS, United Kingdom.

${ }^{2}$ Centre for Hybrid and Organic Solar Energy (CHOSE), University of Rome Tor Vergata, via del Politecnico 1, Rome 00133, Italy.

${ }^{3}$ Cavendish Laboratory, University of Cambridge, JJ Thompson Avenue, Cambridge CB3

OHE, United Kingdom.

Present Address: § Institut Photovoltaïque d'Île-de-France (IPVF) 30, Route départemental 128, 91120 Palaiseau, France.

\section{Corresponding Author}

*stefania.cacovich@ipvf.fr (S.C.); *gd322@cam.ac.uk (G.D.).

Keywords: hybrid perovskite, STEM-EDX, multivariate analysis, chemical composition, big data, nanoscale

\begin{abstract}
The local chemical composition of halide perovskites is a crucial factor in determining their macroscopic properties and their stability. While the combination of scanning transmission electron microscopy (STEM) and energy-dispersive X-ray spectroscopy (EDX) is a powerful and widely used tool for accessing such information, electron-beam-induced damage and complex formulation of the films make this investigation challenging. Here we demonstrate how multivariate analysis - including statistical routines derived from "big data" research, such as Principal Component Analysis, PCA - can be used to dramatically improve the signal recovery from fragile materials. We also show how a similar decomposition algorithm (Non-negative Matrix Factorisation, NMF) can unravel elemental composition at the nanoscale in perovskite films, highlighting the presence of segregated species and identifying the local stoichiometry at the nanoscale.
\end{abstract}


Research into hybrid organic-inorganic perovskite solar cells (PSCs) has flourished over recent years, attracting strong interest by the scientific community ${ }^{1-3}$. This emerging class of devices has become increasingly popular due to the opportunity of reaching good power conversion while being compatible with wet chemistry processing for large area devices. The global research effort associated with the rise of hybrid perovskites resulted in rapid advances in chemical formulation ${ }^{4-}$ ${ }^{7}$, fabrication methods ${ }^{8-10}$ and device architecture ${ }^{11,12}$ - however, most of the progress has been through empirical device improvements, and a number of key questions still remain unanswered. Open issues are covered in reviews ${ }^{13,14}$ and include the optimal chemical composition of the perovskite films ${ }^{11}$, ion migration ${ }^{15,16}$, scalable fabrication routes ${ }^{17,18}$, device architecture ${ }^{19}$ and stability in operation $^{20}$. Specifically, long term stability of the modules under operating conditions is considered to be the main drawback preventing commercial applications ${ }^{21}$. However, poor stability not only prevents easy commercialisation - it also complicates scientific research: commonly used optical and analytical characterisation tools can induce reversible or irreversible structural/chemical changes in the perovskite films through the use of high energy photon or electron beams ${ }^{22-25}$.

In this work we propose an approach that combines the acquisition of high resolution chemical maps by scanning transmission electron microscopy (STEM) with dedicated MultiVariate Analysis (MVA) ${ }^{26}$ methods that improve signal/noise ratio (SNR) and identify correlations between the spatial distribution of elements. Such correlations emerge from statistical analysis, and consist of maps that describe the distribution of chemical compounds rather than just elements. The use of methods that minimise operator input improves the reproducibility of results and the sensitivity to unexpected chemical compounds, such as phases with unpredicted stoichiometry, or elemental segregation. This approach is particularly valuable for hybrid perovskite-based films and devices, in which complex compounds can form, ionic species are prone to migration, and the electron dose during STEM analysis needs to be minimised to prevent local damage.

Specifically, we tested and compared different computational methods - Principal Component Analysis (PCA) ${ }^{27}$ and Non-Negative Matrix Factorisation $(\mathrm{NMF})^{28}$, demonstrating how they can be used to increase the SNR ratio of a dataset and provide new insights on the local chemistry.

The process flow of the paper is as follows: Initially, a cross-sectional sample is extracted from a solar cell (or a perovskite film) using conventional Focused Ion Beam (FIB) preparation ${ }^{29}$ and transferred to a (scanning) transmission electron microscope (STEM). STEM-EDX (Energy Dispersive X-ray spectroscopy) analysis is carried out using optimised illumination conditions to limit the electron dose on the sample, and the experimental data is processed using MVA algorithms available in open source scientific analysis packages such as python-based Hyperspy ${ }^{30}$.

SEM, TEM and STEM are widely used techniques for nanomaterials characterisation. The electron beam, however, can cause temporary or permanent changes in the sample. Such changes can be related to local charging, heating, chemical reactions, preferential removal of atomic species or deposition of carbonaceous contamination ${ }^{31}$. These phenomena are dependent on several factors, including the atomic number of the elements in the specimen, their bonding, and the electrical and thermal conductivity of the specimen, as well as the primary beam illumination 
conditions (acceleration voltage, electron flux and dwell time). For analytical STEM, the electron dose can be kept low by minimising exposure time during specimen survey and optimisation of acquisition conditions, and by the use of high efficiency detectors, such as the latest generation silicon drift detectors for $\mathrm{EDX}^{32}$. Over the last few years several studies reported the use of electron microscopy techniques for the characterisation of perovskite nanocrystals ${ }^{33}$, single crystals ${ }^{34}$, thin films ${ }^{35}$ or full devices ${ }^{36,37}$. Due to the extent and complexity of damage mechanisms that can be triggered in hybrid perovskites during chemical mapping, the signal-to-noise ratio is limited by the onset of degradation - hence the need for advanced data processing algorithms, such as the MVA routines outlined here.

Fast detectors and inexpensive processing power in electron microscopy are allowing the collection of large datasets, which can be treated using approaches developed for "big data" 38 . Multivariate analysis provides a suite of powerful mathematical tools to organise and structure complex and noisy data ${ }^{26}$, widely employed in the treatment of multidimensional datasets ${ }^{39,40}$. Whereas conventional signal analysis (for EDX or EELS, for example) treats each spectrum independently, MVA leverages the total information contained in a large dataset, leading to the separation of signal from noise and the identification of correlations between features. Here, we use and compare different decomposition algorithms for multivariate analysis, all integrated in an open source python-based suite, HyperSpy ${ }^{30}$. HyperSpy provides tools to facilitate interactive data analysis of multidimensional datasets, e. g. EDX or EELS spectrum images. A Spectrum Image (SI) comprises a set of spectra (one, or more, per pixel); such dataset can be decomposed as the linear combination of a set of factors, weighed by a set of corresponding loadings. The analysis of factors (the eigenvalues) and loadings (the eigenvectors), together referred to as "components", can be carried out with a variety of methods, differing by the constraints applied to the dataset. In general, features that are spatially correlated (such as, in EDX, characteristic peaks for different elements in a given compound) will be described by the same component. Thus, for each component of the signal, the factor highlights the correlations between elemental species identifying, for example, different stoichiometries - while the loading map indicates where such elements are located. In this work we employ two of the most commonly used algorithms for data denoising and analysis - PCA and NMF. More details on PCA and NMF algorithms can be found in the SI.

The solar cell under investigation is shown in cross section in Figure 1. The device architecture consists of: FTO (fluorine doped tin oxide) / compact $\mathrm{TiO}_{2} /$ mesoporous $\mathrm{TiO}_{2} / \mathrm{CH}_{3} \mathrm{NH}_{3} \mathrm{PbI}_{3} /$ Spiro-OMeTAD / gold, where the $\mathrm{CH}_{3} \mathrm{NH}_{3} \mathrm{PbI}_{3}$ perovskite layer was prepared using the solvent engineering method ${ }^{41}$. A cross section (lamella) of the device was cut using focused ion beam (FIB) milling and moved to a TEM compatible mount, where it was thinned to $200 \mathrm{~nm}$ by further ion milling in a procedure that has been reported by several groups for metal halide perovskite full devices $^{35,42,43}$. In order to investigate possible damage during sample preparation or TEM analysis, Jeangros et al. thinned the lamellae to different thicknesses, ranging from $<100 \mathrm{~nm}$ to $>500 \mathrm{~nm}^{42}$. A final lamella thickness of about $200 \mathrm{~nm}$ was chosen, as thinner samples are prone to degradation to $\mathrm{PbI}_{2}$ during the FIB step ${ }^{42}$. Moreover, to reduce the interaction with moisture the transfer air 
exposure from the FIB to the TEM should be kept below 5 minutes. The integrity of the sample after FIB preparation was also proved via $\mathrm{XRD}^{44}$ or comparing samples prepared with and without FIB, as well as by varying the TEM exposure time ${ }^{36}$. Overall, the scientific literature has thus validated the use of FIB for preparing TEM samples from perovskite films/devices.

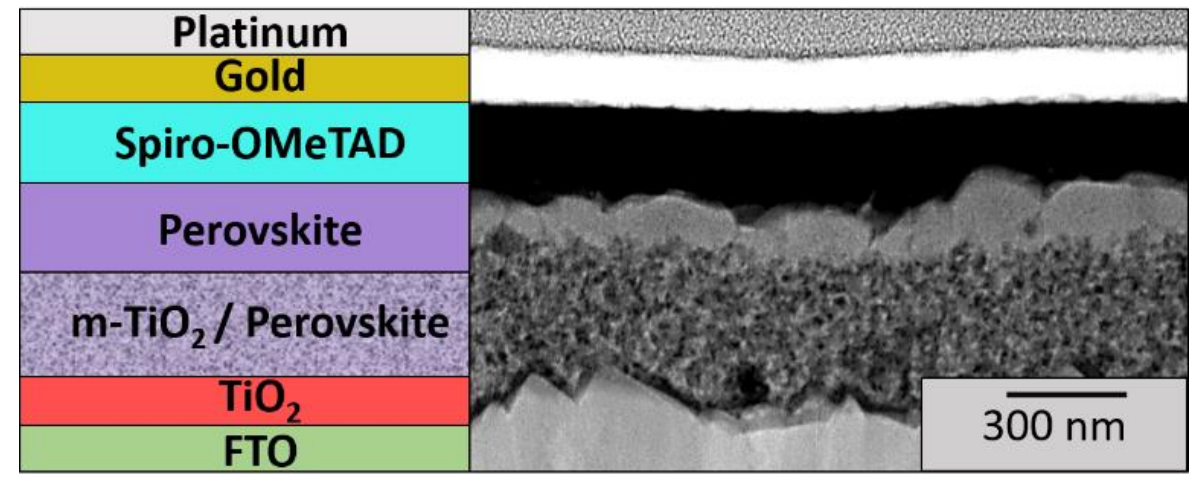

Figure 1. STEM-HAADF image of a perovskite solar cell. From top: platinum, top electrode (gold), hole transport material (Spiro-OMeTAD), active layer (methylammonium lead iodine perovskite - $\mathrm{CH}_{3} \mathrm{NH}_{3} \mathrm{PbI}_{3}$ ), mesoporous titania-perovskite (fluorine doped tin oxide), titania blocking layer, bottom electrode (FTO).

We first acquired an EDX map of the device, with dwell time of $100 \mathrm{~ms}$ and spatial sampling every $10 \mathrm{~nm}$, using a $600 \mathrm{pA}$ current in a $0.5 \mathrm{~nm}$ probe. PCA decomposition was initially used to separate detectable signal from the noise level. As PCA orders the components in order of associated variance within the dataset (as shown in Figure 2), high-order components only model noise - that is, local variation with low correlation with the rest of the information. A denoised model of the dataset can thus be built by employing just the components that contain physically significant signal, discarding the components that describe random noise. This raises the crucial issue of how to determine the noise level in the PCA decomposition.

The dimensionality of the data (and thus the noise level) can be observed by plotting the explained variance ratio against the component number in a logarithmic y scale. This plot, usually referred to as "scree plot" 45 , shows a rapid decrease in the case of sparse data, such as the signal in electron microscopy, and a slow decrease for random noise. The point where the gradient of the explained variance ratio drops is considered a good estimate of the dimensionality of the dataset ${ }^{46}$. For the decomposition shown in Figure 2, a model containing physical information can be built considering the first six components. Choosing the correct number of components can be challenging, particularly in the case of small or highly localised variations that might be producing a variance that is comparable with the noise level. Underestimating the number of the relevant components removes part of the signal, while overestimating it leads to non-optimal denoising. In Figure 2 the factors and loadings for the first seven components are shown, highlighting the noiselike nature of the seventh and successive components. In the loading maps, the colourscale 
represents the weight of the corresponding factor required to fit the original signal at each given pixel. Some components, such as number 6, model EDX features from several elements, highlighting a compound (in this case perovskite, identifiable both from the peak positions in the factor and from the spatial distribution in the loading map). Other components, such as number 1 and , model just one element - gold and carbon in this case- and hence the corresponding loading maps resemble conventional elemental maps. Components 2 and 5 do not have a simple chemical interpretation, but are necessary to account for the variance in the statistical dataset.

\section{PCA Scree Plot Signal}

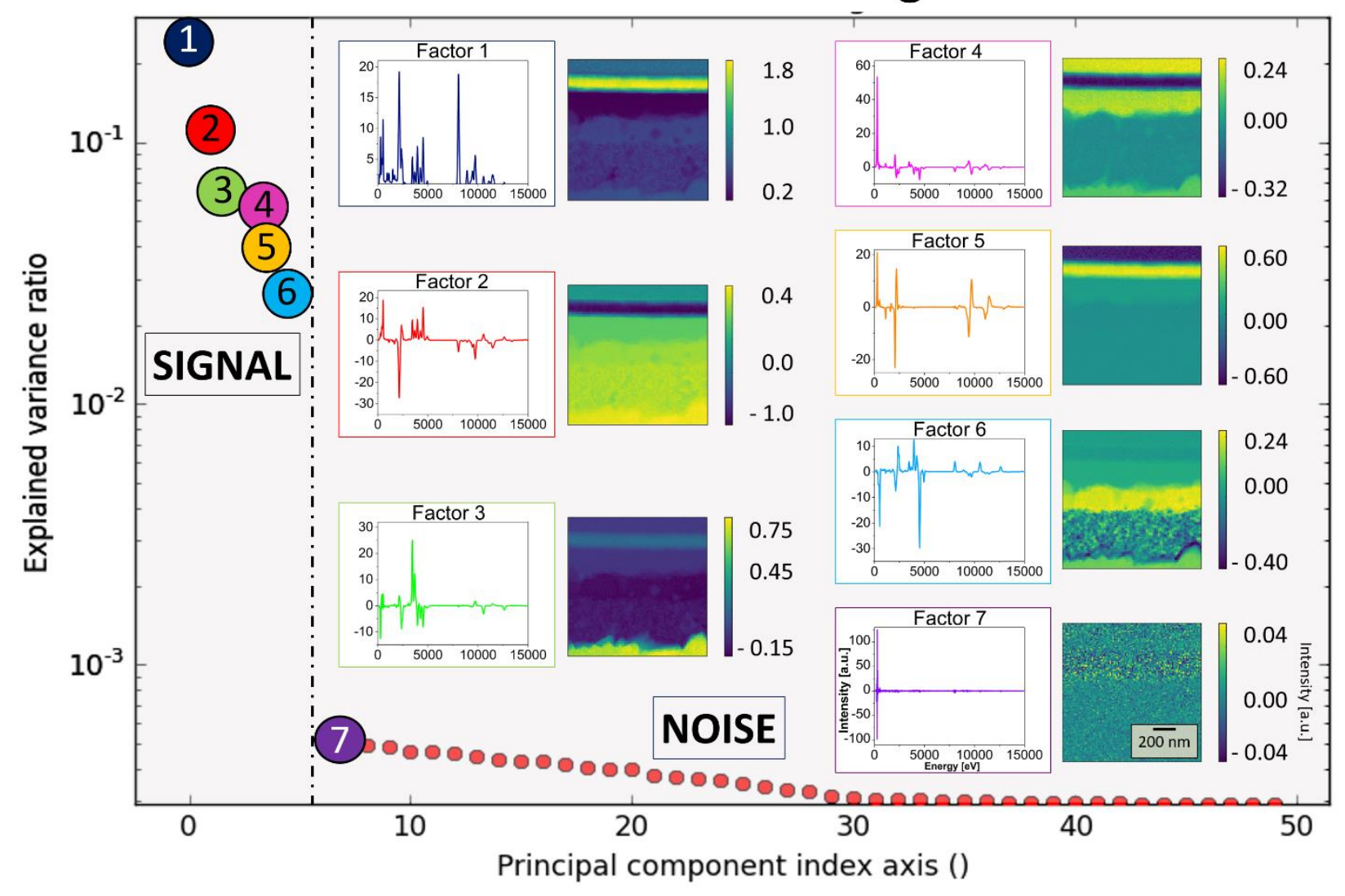

Figure 2. Scree plot and first seven components from a PCA decomposition of the EDX spectrum image (Inset). Each factor is plotted and associated to a spatial map (loading) and is colour-coded to the datapoint on the explained variance scale. The colourscale intensity indicates the weight of the factor for a given pixel.

A denoised spectrum at each pixel can be generated by combining the principal components, with their factors and loadings relevant to the specific pixel. This is directly comparable to the raw data, but with enhanced SNR. Figure 3 reports an example of the improvement in signal quality obtained through PCA denoising. We show raw data (Figure 3a) and a denoised spectrum (Figure $3 b)$ reconstructed from the first six PCA components of spectrum images acquired in other regions of the sample. These two spectra correspond to the blue pixel of Figure 3d. Considering, for 
instance, the $\mathrm{Pb} \mathrm{M}$ peak at $2342 \mathrm{eV}$, the SNR in the raw dataset (100 ms dwell time) and in the denoised spectrum was found to be 4 and 10, respectively - a SNR ratio increase by a factor of 2.5. For comparison, a spectrum acquired in an equivalent area of the same device (red box - Figure $3 \mathrm{~d})$ with a longer dwell time $(10 \mathrm{x})$ is displayed in Figure 3c $(\mathrm{SNR}=8)$. In EDX analysis, the detectability of a peak can be determined by using the Rose criterion, according to which a signal is detectable when SNR $>3{ }^{47}$. In both cases - denoised vs. long acquisition - EDX characteristic peaks are easy identifiable. This comparison also serves as a validation of the denoising process, since all the peaks match in position and relative intensity, and the denoising process does not introduce spurious features. By adopting this approach to data analysis it is possible to

reduce the dwell time and/or the beam current (and hence the electron dose) required for effective EDX mapping, while still obtaining high quality datasets. It is worth noting that a good signal-to-noise ratio is essential for quantitative analysis which can be later performed on the denoised spectra ${ }^{48,49}$.

Improvements in the analysis of EDX data allow the acquisition of datasets at a reduced electron dose, which is often a limiting factor for anything but the most robust samples. To explore the limit in electron dose in our example, we acquired a series of spectrum images on equivalent areas of the specimen (i.e. similar thickness) by varying both dwell time and defocus value. Varying the focusing conditions implies a variation in the probe size, as explained in fig S1. A series of EDX spectrum images was acquired using two different dwell times, namely 1 (red) and $100 \mathrm{~ms}$ (orange), and going overfocus $(+500 \mathrm{~nm},+1000 \mathrm{~nm})$, underfocus $(-500 \mathrm{~nm},-1000 \mathrm{~nm})$ and in focus, as shown in Fig 3e. The probe size was calculated to be approximately $0.5 \mathrm{~nm}$ when in focus, $1.3 \mathrm{~nm}$ for $\pm 500 \mathrm{~nm}$ out of focus and $2.6 \mathrm{~nm}$ for \pm 1000 out of focus. In the case of long dwell time the HAADF images show that the lamella is clearly damaged, with an evident morphological degradation. Conversely, a dwell time of $100 \mathrm{~ms}$ has not induced any visible degradation in the perovskite active layer. 

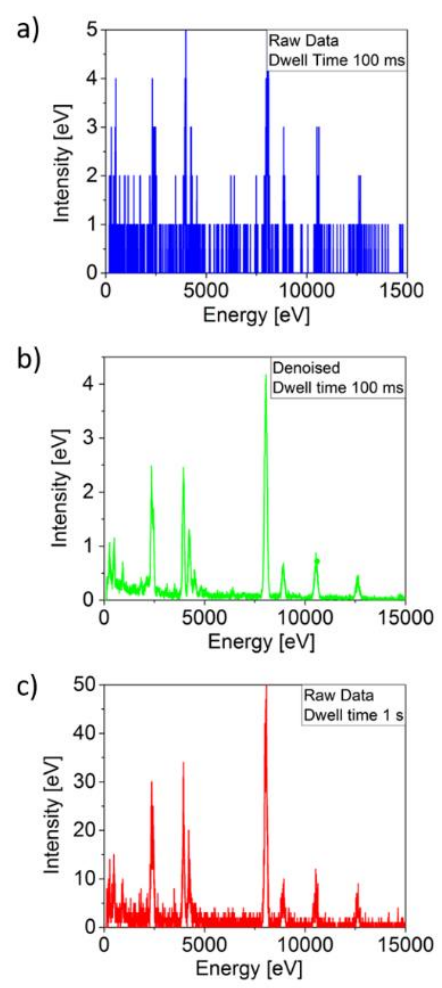

d)

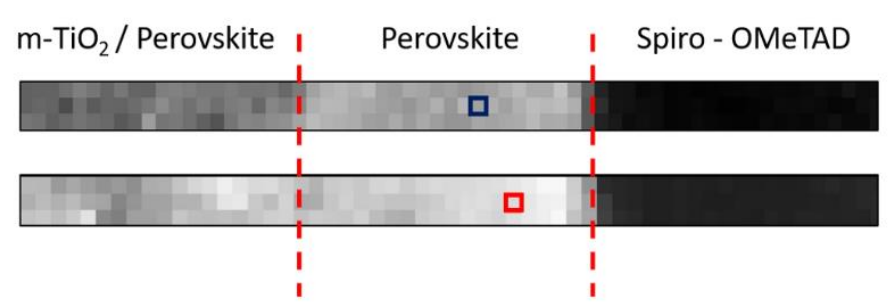

e)

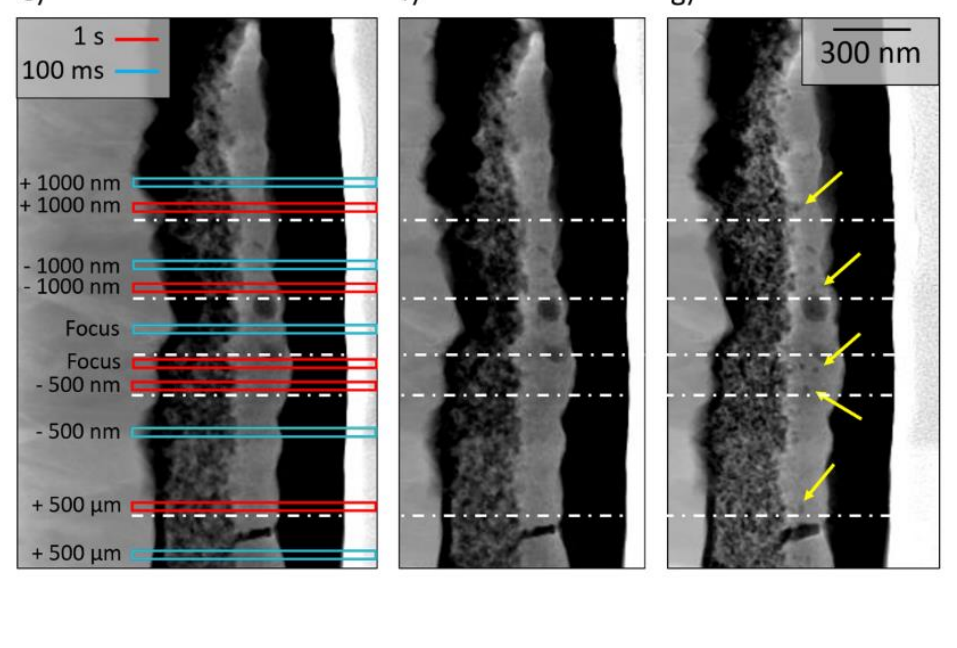

Figure 3. Example of denoised spectrum. Original spectrum (a), denoised spectrum (b) and spectrum acquired with a longer dwell time (c). Spectra (a) and (b) refer to the blue pixel in the spectrum image(d), whereas spectrum (c) is extracted from the red pixel. The pixel size is $10 \mathrm{~nm}$. Dwell time and focus were changed to test the possible effects of the beam on the sample(e). STEM-HAADF images before (f) and after (g) EDX spectra acquisition. Visible morphological changes are highlighted by the yellow arrows. 


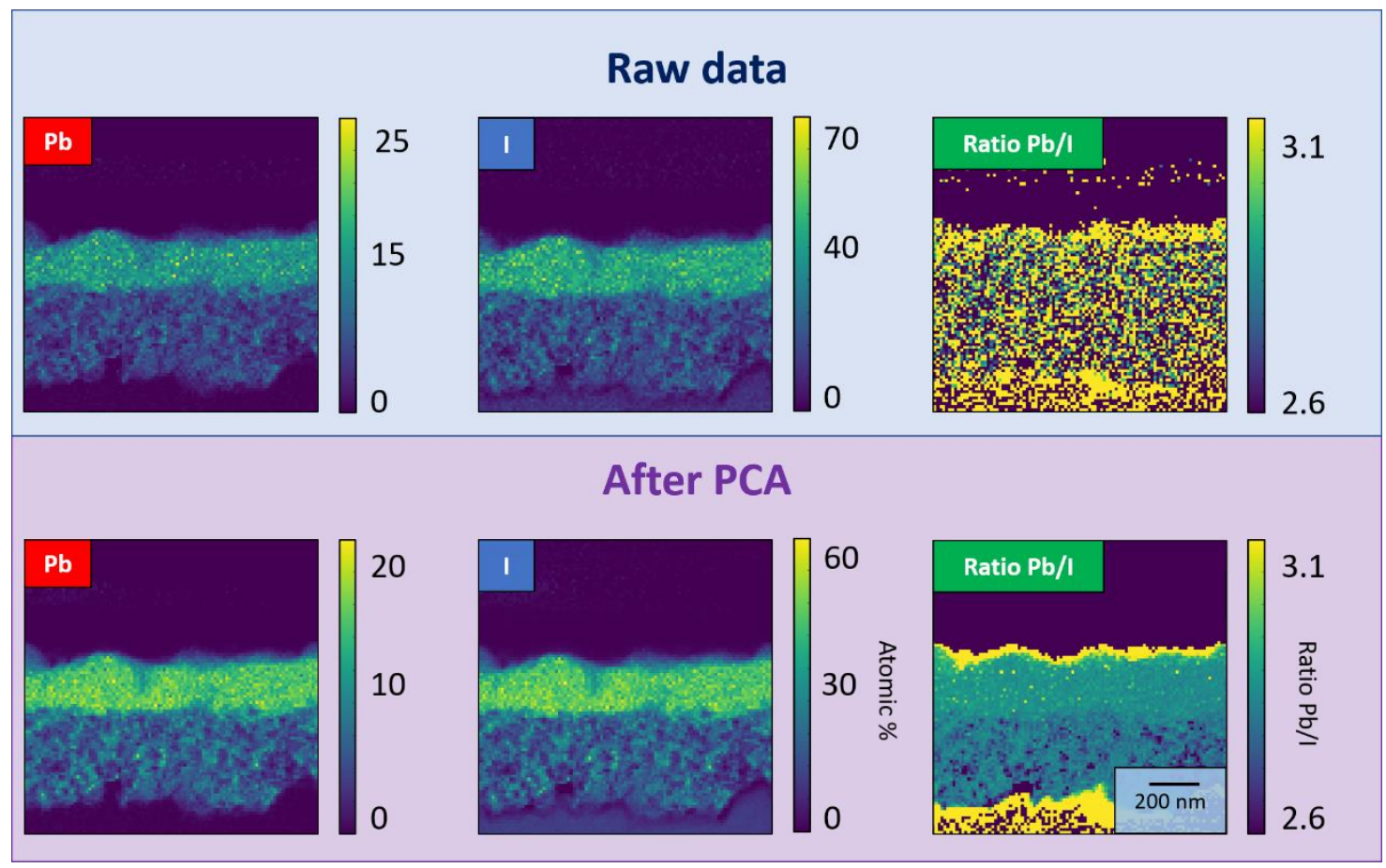

Figure 4. Lead and iodine quantitative maps calculated on the raw data and after PCA denoising routine and corresponding ratio between the two elements.

The benefit of the application of PCA to EDX datasets is highlighted in Fig 4, where the Pb/I elemental ratio, a crucial parameter in the study of halide perovskites, was investigated. While the ratio calculated from the raw data appears noisy and with no physical meaning, the maps obtained after denoising describes in detail the morphology and the local chemical variations layer by layer. The level of perovskite infiltration in the mesoporous layer is well defined and the ratio is almost constant in the perovskite compact layer, suggesting that the electron beam has not induced any change in the material. The apparent iodine presence in the FTO is due to a peak overlap of I $\mathrm{L}_{\alpha}$ and $\mathrm{Sn} \mathrm{L}_{\beta}$, which is very challenging to deconvolute. Elemental maps for the all the elements before and after PCA denoising are reported in S2 and S3, respectively.

While PCA is perhaps the most common MVA algorithm, other decomposition methods can be applied to EDX data by imposing different conditions on the factors. In some cases, more meaningful physical information can be obtained using, for example, NMF. NMF further increases the constraints on the components, imposing non-negativity, resulting in a sparse and part-based representation, which is more interpretable than a PCA decomposition. The obtained components (Figure 5) are more directly connected to the physical signal (EDX counts are non-negative) and the contrast in the maps is higher due to the reduced correlation signal. NMF is a more computationally intensive algorithm compared to PCA, requiring 10-100 times longer to compute. NMF is thus very useful for analysis and interpretation, but PCA is very well suited to initial screening of data and denoising. In Figure 5, we can clearly identify the different layers comprising 
the device: gold (factor 1), perovskite (factor 2), FTO (factor 3), Spiro-OMeTAD (factor 4), platinum (factor 5) and titania (factor 6). A good contrast can be seen for example showing the infiltration of the perovskite inside the titania scaffold, evident from components 2 and 6 . We can note that the NMF algorithm allows also to overcome the issues related to peak overlap. In this case tin and iodine signals are separated in components representing perovskite and FTO respectively.

\section{Factor 1}

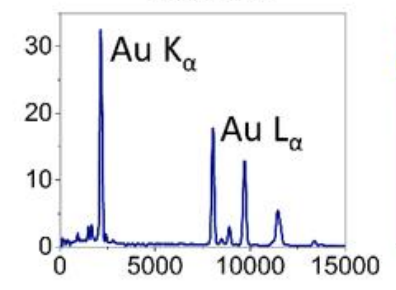

Factor 3

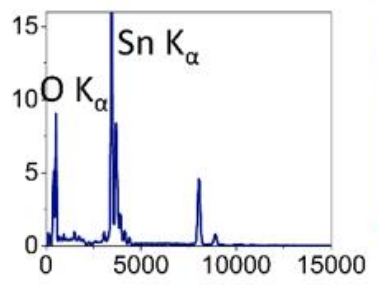

Factor 5

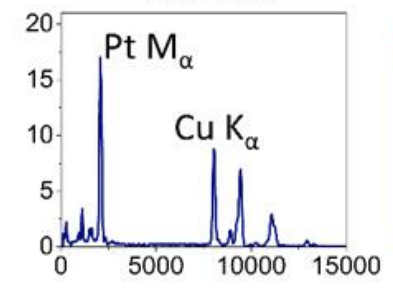

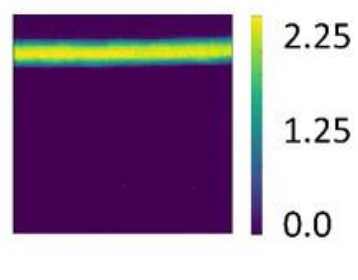
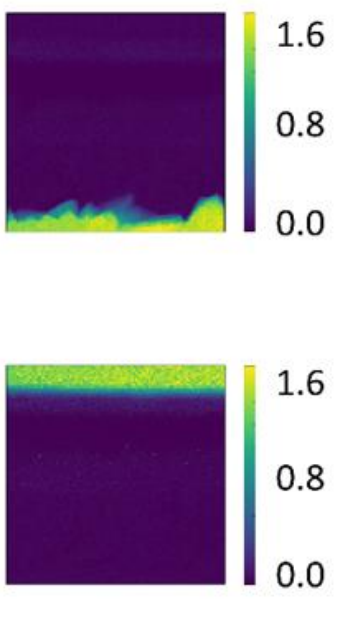

Factor 2

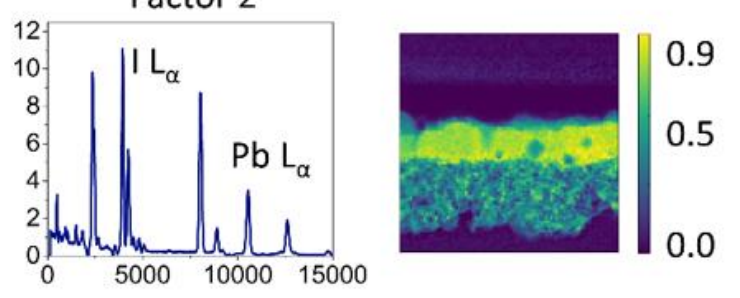

Factor 4
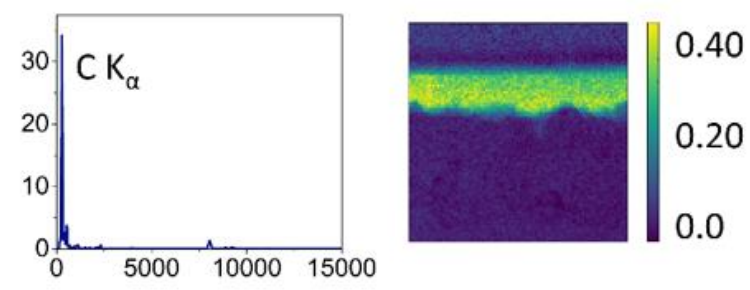

Factor 6
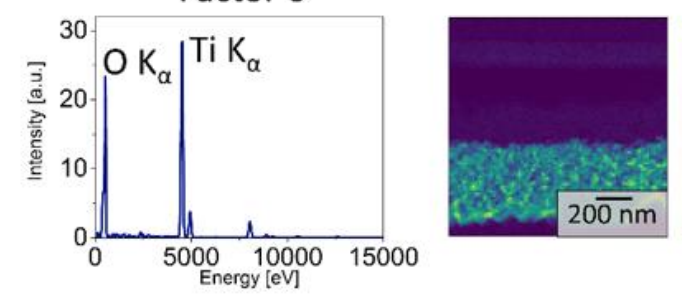

0.72

0.4

Figure 5. NMF decomposition results on the same dataset analysed in Figure 2. Different compounds are differentiated and identified from their EDX features. The loading of the corresponding component represents the spatial distribution of the compound / phase in the sample.

PCA is therefore very suited to data denoising, using the scree plot as a simple and intuitive tool to determine the dimensionality of the dataset, also thanks to its short computation time (seconds on an office desktop PC for 100 x 100 pixels $^{2}$ x 4000 values). After determining the physically significant components, NMF can be applied to extract information with high contrast and straightforward physical interpretation, with each compound identified by a specific spatial distribution $^{50}$, at the cost of a longer processing time. One of the main drawbacks of PCA decomposition is that the output signals are not always easy to interpret because they are often characterised by the presence of strong negative peaks. Negative values in the factors are generated by anti-correlation in the spectral features, and it is important to note that these factors are not 
EDX spectra (negative counts being non-physical), but rather components for a model of an EDX dataset.

The chemistry of hybrid perovskites in state-of-the-art devices is now quite complex and includes the presence of three monovalent cations (typically Cs, FA and MA), lead, and one or more halides ${ }^{4}$. Additionally, recently published papers proved that both the stability and the optoelectronics properties of these materials strongly benefit from the addition of a small quantity of monovalent cations such as sodium, potassium or rubidium ${ }^{49-53}$. NMF provides a tool for identifying local stoichiometric variations in a process that requires minimal operator bias, allowing chemical information to emerge from correlated elemental distributions. To demonstrate the benefits of NMF of STEM-EDX data, we processed a dataset from a $\mathrm{Cs}_{0.05} \mathrm{FA}_{0.78} \mathrm{MA}_{0.17} \mathrm{~Pb}\left(\mathrm{I}_{0.85} \mathrm{Br}_{0.15}\right)_{3}$ thin film passivated with potassium halide interlayers arising from the addition of $\mathrm{KI}$ to the perovskite precursor solution. In particular, we denote the fraction of $\mathrm{K}$ out of the $\mathrm{A}$ site as $\mathrm{x}=[\mathrm{K}] /([\mathrm{A}]+[\mathrm{K}])$ and $\mathrm{A}=\mathrm{Cs}_{0.05} \mathrm{FA}_{0.78} \mathrm{MA}_{0.17}$. Figure 6 shows a STEM high angle annular dark field (HAADF) cross sectional view of a perovskite thin film with $\mathrm{x}=0.2$ lamella. To preserve the perovskite layer during specimen preparation, we deposited SpiroOMeTAD and platinum as capping layers. NMF leads to the identification of two phases within the active layer, labelled as factor 1 and factor 2 in Figure $6 \mathrm{~d}$ and 6e, respectively. Factor 1 shows $\mathrm{Br} \mathrm{L} \alpha, \mathrm{Pb} \mathrm{M \alpha}, \mathrm{I} \mathrm{L}_{\alpha}$ characteristic EDX peaks, which can be associated with the perovskite phase. Factor 2 is representative of a second phase - rich in bromine and potassium - with a different stoichiometry. Interestingly, the signal linked to factor 2 is particularly strong at the grain boundaries of the perovskite thin film. This suggests the formation of a particularly $\mathrm{Br}$ - and K-rich passivation layer decorating the crystalline surfaces including grain boundaries, top and bottom interfaces. In this case the finding was confirmed by other techniques and shown to be directly related to the net improvement of the luminescence and charge transport ${ }^{6}$. For comparison, EDX elemental maps of bromine and potassium are shown in Fig $6 \mathrm{f}$ and $6 \mathrm{~g}$. The quantification was performed on the raw EDX datasets, demonstrating that traditional EDX maps are not able to unveil the formation of the passivation layer at the grain boundaries. Quantitative maps for all elements are reported in S4. 

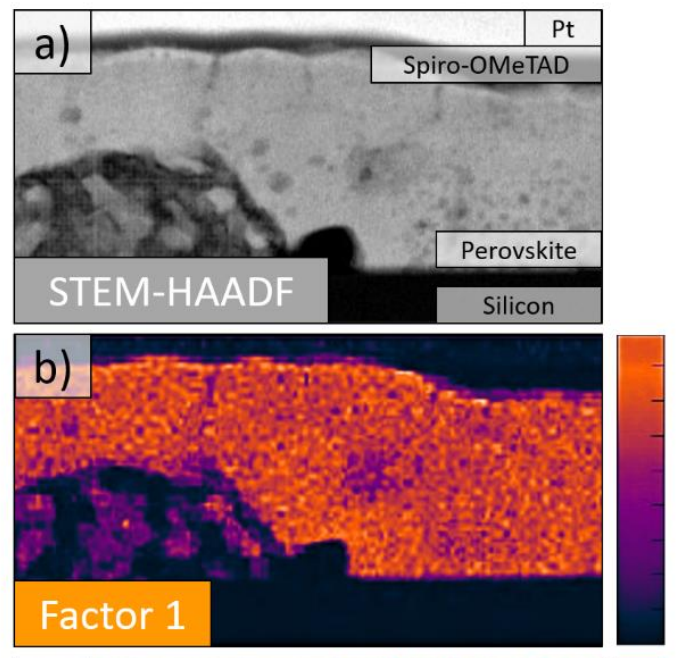

0.16
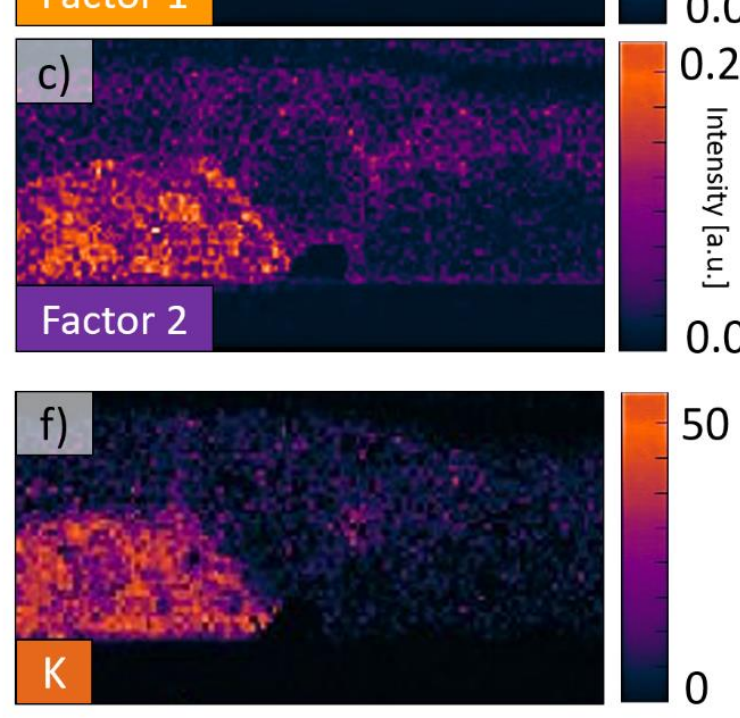
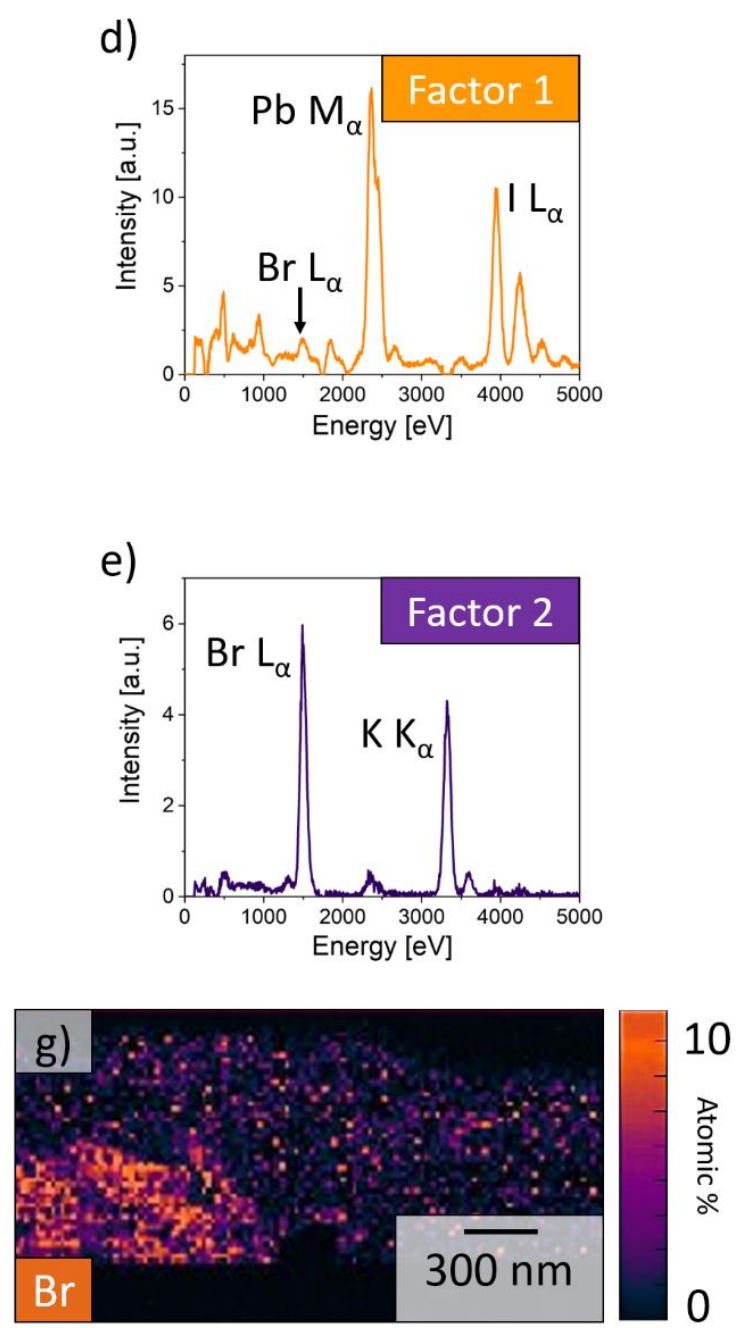

Figure 6. STEM-HAADF cross sectional image of a perovskite thin film ${ }^{6}$ with potassium concentration of $x=0.20$ (a). NMF decomposition results in factor 1 (spatial distribution in panel $\mathrm{b}$, profile in panel d) associated to the perovskite layer and in factor 2 (spatial distribution in panel c, profile in panel e) indicating the presence of a potassium- and bromine-rich phase. Quantitative EDX elemental maps denoising for $\mathrm{K}(\mathrm{f})$ and $\mathrm{Br}(\mathrm{g})$.

Obtaining information from chemical maps at the nanoscale from complex, beam-sensitive materials such as hybrid perovskites is a major challenge. In this study we demonstrate how STEM-EDX data analysis can be refined using multivatiate statistical methods, which assist in minimising the electron dose for efficient elemental mapping and in identifying different chemical phases. The analysis can be carried out with custom-written scripts or using widely available open source packages developed by the scientific community, such as Hyperspy. Efficient data processing can be performed employing PCA for data denoising, and NMF for determining the different compounds and phases present in a specific material, or for the identification of the 
different layer or features within the solar cells. The same analytical approach can be used in the investigation of novel complex compounds, with particular benefits in materials and devices that are beam-sensitive or contain a variety of intermixed compounds. Additionally, decomposition can be a powerful tool in the investigation of dynamic processes - for example, for in situ measurements in the TEM: denoising routines allow the extraction of information from data acquired quickly and with poor SNR, whereas factorisation can directly track the emergence of new phases.

\section{Conflict of interest}

There are no conflicts of interest to declare.

\section{Associated Content}

The supporting information includes detailed methods on Device Fabrication, Thin Film Fabrication and Electron Microscopy Characterisation.

\section{Acknowledgments}

S.C., C.D. and G.D. acknowledge funding from ERC under grant number 25961976 PHOTO EM and financial support from the EU under grant number 77312483 ESTEEM2. S.C., C.D. and G.D. also thank Dr. Francisco de la Peña and Dr. Pierre Burdet for very helpful discussions regarding Hyperspy and MVA. The CHOSE team gratefully acknowledges the European Union's Horizon 2020 Framework Program for funding Research and Innovation under Grant agreement no. 653296 (CHEOPS). M.A.-J. thanks Nava Technology Limited, Cambridge Materials Limited and EPSRC (grant number: EP/M005143/1) for their funding and technical support. S.D.S. acknowledges support from the Royal Society and Tata Group (UF150033) and the European Research Council (ERC) under the European Union's Horizon 2020 research and innovation programme (grant agreement number 756962).

\section{References}

(1) Snaith, H. J. Present Status and Future Prospects of Perovskite Photovoltaics. Nat. Mater. 2018, 17, 372-376.

(2) Stranks, S. D.; Snaith, H. J. Metal-Halide Perovskites for Photovoltaic and Light-Emitting Devices. Nat. Nanotechnol. 2015, 10, 391-402.

(3) Green, M. A.; Ho-Baillie, A.; Snaith, H. J. The Emergence of Perovskite Solar Cells. Nat. Photonics 2014, 8, 506-514.

(4) Saliba, M.; Matsui, T.; Seo, J.-Y.; Domanski, K.; Correa-Baena, J.-P.; Nazeeruddin, M. K.; Zakeeruddin, S. M.; Tress, W.; Abate, A.; Hagfeldt, A.; Graetzle M. Cesium-Containing Triple Cation Perovskite Solar Cells: Improved Stability, Reproducibility and High Efficiency. Energy Environ. Sci. 2016, 9, 1989-1997. 
(5) Saliba, M.; Matsui, T.; Domanski, K.; Seo, J.-Y.; Ummadisingu, A.; Zakeeruddin, S. M.; Correa-Baena, J.-P.; Tress, W. R.; Abate, A.; Hagfeldt, A.; Graetzle M. Incorporation of Rubidium Cations into Perovskite Solar Cells Improves Photovoltaic Performance. Science 2016, 354, 206-209.

(6) Abdi-Jalebi, M.; Andaji-Garmaroudi, Z.; Cacovich, S.; Stavrakas, C.; Philippe, B.; Richter, J. M.; Alsari, M.; Booker, E. P.; Hutter, E. M.; Pearson, A. J.; Lilliu S.; Savenije T.J.; Rensmo H; Divitini G.; Ducati C.; Friend R.H.; Stranks S.D. Maximizing and Stabilizing Luminescence from Halide Perovskites with Potassium Passivation. Nature 2018, 555, 497501.

(7) Zhang, Y.; Grancini, G.; Feng, Y.; Asiri, A. M.; Nazeeruddin, M. K. Optimization of Stable Quasi-Cubic $\mathrm{FA}_{\mathrm{x}} \mathrm{MA}_{1-\mathrm{X}} \mathrm{PbI}_{3}$ Perovskite Structure for Solar Cells with Efficiency beyond 20\%. ACS Energy Lett. 2017, 2, 802-806.

(8) Jeon, N. J.; Noh, J. H.; Yang, W. S.; Kim, Y. C.; Ryu, S.; Seo, J.; Seok, S. I. Compositional Engineering of Perovskite Materials for High-Performance Solar Cells. Nature 2015, 517, 476-480.

(9) Jeon, N. J.; Noh, J. H.; Kim, Y. C.; Yang, W. S.; Ryu, S.; Seok, S. I. Solvent Engineering for High-Performance Inorganic-Organic Hybrid Perovskite Solar Cells. Nat. Mat. 2014, 13, 897-903.

(10) Stranks, S. D.; Nayak, P. K.; Zhang, W.; Stergiopoulos, T.; Snaith, H. J. Formation of Thin Films of Organic-Inorganic Perovskites for High-Efficiency Solar Cells. Angew. Chem. 2015, 54, 3240-3248.

(11) Seo, J.-Y.; Uchida, R.; Kim, H.-S.; Saygili, Y.; Luo, J.; Moore, C.; Kerrod, J.; Wagstaff, A.; Eklund, M.; McIntyre, R.; Pellet, N.; Zakeeruddin S.K.; Hagfeldt A.; Graetzle M. Boosting the Efficiency of Perovskite Solar Cells with CsBr-Modified Mesoporous $\mathrm{TiO}_{2}$ Beads as Electron-Selective Contact. Adv. Funct. Mat. 2018, 28, 1705763.

(12) Abate, A.; Correa-Baena, J.-P.; Saliba, M.; Suait, M. S.; Bella, F. Perovskite Solar Cells: From the Laboratory to the Assembly Line. Chem. Eur. J. 2018, 24, 3083-3100.

(13) Correa-Baena, J.-P.; Saliba, M.; Buonassisi, T.; Grätzel, M.; Abate, A.; Tress, W.; Hagfeldt, A. Promises and Challenges of Perovskite Solar Cells. Science 2017, 358, 739-744.

(14) Correa-Baena, J.-P.; Abate, A.; Saliba, M.; Tress, W.; Jesper Jacobsson, T.; Grätzel, M.; Hagfeldt, A. The Rapid Evolution of Highly Efficient Perovskite Solar Cells. Energy Environ. Sci. 2017, 10, 710-727.

(15) Snaith, H. J.; Abate, A.; Ball, J. M.; Eperon, G. E.; Leijtens, T.; Noel, N. K.; Stranks, S. D.; Wang, J. T.-W.; Wojciechowski, K.; Zhang, W. Anomalous Hysteresis in Perovskite Solar Cells. J. Phys. Chem. Lett. 2014, 5, 1511-1515.

(16) Tress, W.; Correa Baena, J. P.; Saliba, M.; Abate, A.; Graetzel, M. Inverted Current-Voltage Hysteresis in Mixed Perovskite Solar Cells: Polarization, Energy Barriers, and Defect Recombination. Adv. Energy Mater. 2016, 6, 1600396.

(17) Razza, S.; Di Giacomo, F.; Matteocci, F.; Cinà, L.; Palma, A. L.; Casaluci, S.; Cameron, P.; D’Epifanio, A.; Licoccia, S.; Reale, A.; Brown, T.M.; Di Carlo, A. Perovskite Solar Cells and Large Area Modules $\left(100 \mathrm{Cm}^{2}\right)$ Based on an Air Flow-Assisted $\mathrm{PbI}_{2}$ Blade Coating Deposition Process. J. Power Sources 2015, 277, 286-291.

(18) Kim, J.; Yun, J. S.; Cho, Y.; Lee, D. S.; Wilkinson, B.; Soufiani, A. M.; Deng, X.; Zheng, J.; Shi, A.; Lim, S.; Chen, S. Hameiri, Z.; Zhang, M.; Lau, C.F.J; Huang, S.; Green, M.A.; Ho-Baillie A.W.Y. Overcoming the Challenges of Large-Area High-Efficiency Perovskite Solar Cells. ACS Energy Lett. 2017, 2, 1978-1984. 
(19) Futscher, M. H.; Ehrler, B. Modeling the Performance Limitations and Prospects of Perovskite/Si Tandem Solar Cells under Realistic Operating Conditions. ACS Energy Lett. 2017, 2, 2089-2095.

(20) Leijtens, T.; Eperon, G. E.; Noel, N. K.; Habisreutinger, S. N.; Petrozza, A.; Snaith, H. J. Stability of Metal Halide Perovskite Solar Cells. Adv. Energy Mater. 2015, 5, 1500963.

(21) Berhe, T. A.; Su, W.-N.; Chen, C.-H.; Pan, C.-J.; Cheng, J.-H.; Chen, H.-M.; Tsai, M.-C.; Chen, L.-Y.; Dubale, A. A.; Hwang, B.-J. Organometal Halide Perovskite Solar Cells: Degradation and Stability. Energy Environ. Sci. 2016, 9, 323-356.

(22) Xiao, C.; Li, Z.; Guthrey, H.; Moseley, J.; Yang, Y.; Wozny, S.; Moutinho, H.; To, B.; Berry, J. J.; Gorman, B.; Yan, Y.; Zhu, K.; Al-Jassim, M. Mechanisms of Electron-BeamInduced Damage in Perovskite Thin Films Revealed by Cathodoluminescence Spectroscopy. J. Phys. Chem. C 2015, 119, 26904-26911.

(23) Yuan, H.; Debroye, E.; Janssen, K.; Naiki, H.; Steuwe, C.; Lu, G.; Moris, M.; Orgiu, E.; Uji-i, H.; De Schryver, F.; Samori, P.; Hofkens J.; Roeffaers M. Degradation of Methylammonium Lead Iodide Perovskite Structures through Light and Electron Beam Driven Ion Migration. J. Phys. Chem. Lett. 2016, 7, 561-566.

(24) Klein-Kedem, N.; Cahen, D.; Hodes, G. Effects of Light and Electron Beam Irradiation on Halide Perovskites and Their Solar Cells. Acc. Chem. Res. 2016, 49, 347-354.

(25) Wang, L.; Wang, H.; Wagner, M.; Yan, Y.; Jakob, D. S.; Xu, X. G. Nanoscale Simultaneous Chemical and Mechanical Imaging via Peak Force Infrared Microscopy. Sci. Adv. 2017, 3, e1700255.

(26) Heel, M. van; Portugal, R. V.; Schatz, M. Multivariate Statistical Analysis of Large Datasets: Single Particle Electron Microscopy. Open Journal of Statistics 2016, 06, 701739.

(27) Wold, S.; Esbensen, K.; Geladi, P. Principal Component Analysis. Chemom. Intell. Lab. Syst. 1987, 2, 37-52.

(28) Pauca, V. P.; Piper, J.; Plemmons, R. J. Nonnegative Matrix Factorization for Spectral Data Analysis. Linear Algebra Appl. 2006, 416, 29-47.

(29) Langford, R.; Clinton, C. In Situ Lift-out Using a FIB-SEM System. Micron 2004, 35, 607611.

(30) de la Peña, F.; Ostasevicius, T.; Fauske, V. T.; Burdet, P.; Prestat, E.; Jokubauskas, P.; Nord, M.; Sarahan, M.; MacArthur, K. E.; Johnstone, D. N.; Taillon, J.; Eljarrat, A.; Migunov, V.; Caron, J.; Furnival, T.; Mazzucco, S.; Aarholt, T.; Walls, M.; Tom Slater, T.; Winkler, F.; Martineau B.; Donval G.; McLeod, R.; Hoglund E.R.; Alxneit, I.; Hjorth, I.; Henninen, T.; Zagonel, L.F.; Garmannslund, A. HyperSpy 1.4 (Version v1.1.0+1). Zenodo. http://doi.org/10.5281/zenodo.1408168, 2018.

(31) Egerton, R. F.; Li, P.; Malac, M. Radiation Damage in the TEM and SEM. Micron 2004, 35, 399-409.

(32) Newbury, D. E. X-Ray Spectrometry and Spectrum Image Mapping at Output Count Rates above $100 \mathrm{KHz}$ with a Silicon Drift Detector on a Scanning Electron Microscope. Scanning 2006, 27, 227-239.

(33) Dang, Z.; Shamsi, J.; Palazon, F.; Imran, M.; Akkerman, Q. A.; Park, S.; Bertoni, G.; Prato, M.; Brescia, R.; Manna, L. In Situ Transmission Electron Microscopy Study of Electron Beam-Induced Transformations in Colloidal Cesium Lead Halide Perovskite Nanocrystals. ACS Nano 2017, 11, 2124-2132. 
(34) Dar, M. I.; Jacopin, G.; Hezam, M.; Arora, N.; Zakeeruddin, S. M.; Deveaud, B.; Nazeeruddin, M. K.; Graetzel, M. Asymmetric Cathodoluminescence Emission in $\mathrm{CH}_{3} \mathrm{NH}_{3} \mathrm{PbI}_{3}-\mathrm{XBr}_{\mathrm{x}}$ Perovskite Single Crystals. ACS Photonics 2016, 3, 947-952.

(35) Hentz, O.; Zhao, Z.; Gradečak, S. Impacts of Ion Segregation on Local Optical Properties in Mixed Halide Perovskite Films. Nano Lett. 2016, 16 (2), 1485-1490.

(36) Zhou, Y.; Vasiliev, A. L.; Wu, W.; Yang, M.; Pang, S.; Zhu, K.; Padture, N. P. Crystal Morphologies of Organolead Trihalide in Mesoscopic/Planar Perovskite Solar Cells. $J$. Phys. Chem. Lett. 2015, 6, 2292-2297.

(37) Yang, B.; Dyck, O.; Ming, W.; Du, M.-H.; Das, S.; Rouleau, C. M.; Duscher, G.; Geohegan, D. B.; Xiao, K. Observation of Nanoscale Morphological and Structural Degradation in Perovskite Solar Cells by in Situ TEM. ACS Appl. Mater. Interfaces 2016, 8, 32333-32340.

(38) Jesse, S.; Chi, M.; Belianinov, A.; Beekman, C.; Kalinin, S. V.; Borisevich, A. Y.; Lupini, A. R. Big Data Analytics for Scanning Transmission Electron Microscopy Ptychography. Scientific Reports 2016, 6.

(39) Parish, C. M.; Brewer, L. N. Multivariate Statistics Applications in Phase Analysis of STEM-EDS Spectrum Images. Ultramicroscopy 2010, 110, 134-143.

(40) Kotula, P. G.; Keenan, M. R.; Michael, J. R. Automated Analysis of SEM X-Ray Spectral Images: A Powerful New Microanalysis Tool. Microsc. Microanal. 2003, 9, 1-17.

(41) Matteocci, F.; Cinà, L.; Lamanna, E.; Cacovich, S.; Divitini, G.; Midgley, P. A.; Ducati, C.; Di Carlo, A. Encapsulation for Long-Term Stability Enhancement of Perovskite Solar Cells. Nano Energy 2016, 30, 162-172.

(42) Jeangros, Q.; Duchamp, M.; Werner, J.; Kruth, M.; Dunin-Borkowski, R. E.; Niesen, B.; Ballif, C.; Hessler-Wyser, A. In Situ TEM Analysis of Organic-Inorganic Metal-Halide Perovskite Solar Cells under Electrical Bias. Nano Lett. 2016, 16, 7013-7018.

(43) Conings, B.; Drijkoningen, J.; Gauquelin, N.; Babayigit, A.; D’Haen, J.; D’Olieslaeger, L.; Ethirajan, A.; Verbeeck, J.; Manca, J.; Mosconi, E.; De Angelis, F.; Boyen, H.-G. Intrinsic Thermal Instability of Methylammonium Lead Trihalide Perovskite. Adv. Energy Mater. 2015, 5, 1500477.

(44) Nanova, D.; Kast, A. K.; Pfannmöller, M.; Müller, C.; Veith, L.; Wacker, I.; Agari, M.; Hermes, W.; Erk, P.; Kowalsky, W.; Schroeder, R.R.; Lovrincic R. Unraveling the Nanoscale Morphologies of Mesoporous Perovskite Solar Cells and Their Correlation to Device Performance. Nano Lett. 2014, 14, 2735-2740.

(45) Cattell, R. B. The Scree Test For The Number Of Factors. Multivar. Behav. Res. 1966, 1, 245-276.

(46) Zhu, M.; Ghodsi, A. Automatic Dimensionality Selection from the Scree Plot via the Use of Profile Likelihood. Comput. Stat. Data Anal. 2006, 51, 918-930.

(47) Watanabe, M.; Egerton, R. F. Fundamental Limit to Single-Atom Analysis by STEM-EDX Spectroscopy. Microsc. Microanal. 2017, 23, 380-381.

(48) Divitini, G.; Cacovich, S.; Matteocci, F.; Cinà, L.; Di Carlo, A.; Ducati, C. In Situ Observation of Heat-Induced Degradation of Perovskite Solar Cells. Nat. Energy 2016, 1, 15012.

(49) Cacovich, S.; Ciná, L.; Matteocci, F.; Divitini, G.; Midgley, P. A.; Di Carlo, A.; Ducati, C. Gold and Iodine Diffusion in Large Area Perovskite Solar Cells under Illumination. Nanoscale 2017, 9, 4700-4706. 
(50) Cacovich, S.; Divitini, G.; Ireland, C.; Matteocci, F.; Di Carlo, A.; Ducati, C. Elemental Mapping of Perovskite Solar Cells by Using Multivariate Analysis: An Insight into Degradation Processes. ChemSusChem 2016, 9, 2673-2678.

(51) Duong, T.; Wu, Y.; Shen, H.; Peng, J.; Fu, X.; Jacobs, D.; Wang, E.-C.; Kho, T. C.; Fong, K. C.; Stocks, M.; Franklin, E.; Blakers, A.; Zin, N.; McIntosh, K.; Li, W.; Cheng, Y-B.; White, T.P.; Weber, K.; Catchpole, K. Rubidium Multication Perovskite with Optimized Bandgap for Perovskite-Silicon Tandem with over 26\% Efficiency. Adv. Energy Mater. 2017, 7, 1700228.

(52) Park, Y. H.; Jeong, I.; Bae, S.; Son, H. J.; Lee, P.; Lee, J.; Lee, C.-H.; Ko, M. J. Solar Cells: Inorganic Rubidium Cation as an Enhancer for Photovoltaic Performance and Moisture Stability of $\mathrm{HC}\left(\mathrm{NH}_{2}\right)_{2} \mathrm{PbI}_{3}$ Perovskite Solar Cells. Adv. Funct. Mater. 2017, 27 (16).

(53) Nam, J. K.; Chai, S. U.; Cha, W.; Choi, Y. J.; Kim, W.; Jung, M. S.; Kwon, J.; Kim, D.; Park, J. H. Potassium Incorporation for Enhanced Performance and Stability of Fully Inorganic Cesium Lead Halide Perovskite Solar Cells. Nano Lett. 2017, 17 (3), 2028-2033.

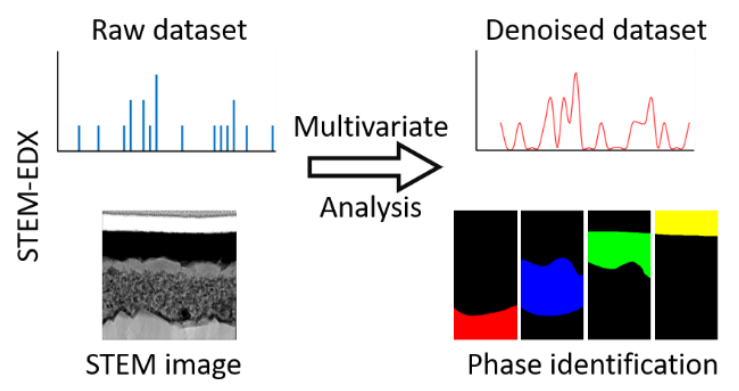

